\title{
AMENAZAS EXÓGENAS Y VULNERABILIDADES ENDÓGENAS \\ TERREMOTOS, CRISIS INTERNACIONALES Y DESARROLLO EN CHILE 1930-2010
}

\section{Oscar LANDERRETCHe GACITÚA (oscar.landerretche@iap.uchile.cl) Instituto de Asuntos Públicos - Universidad de Chile}

Los eventos potencialmente catastróficos de origen natural (como los grandes sismos que afectan al territorio de Chile cada cierto tiempo) tienen en común con las crisis económicas internacionales, su carácter exógeno, desde el punto de vista de una economía abierta y pequeña. En ambos casos, la vulnerabilidad del sistema económico y social está determinada, en gran medida, por la magnitud de los esfuerzos de prevención y de preparación para las emergencias. Estas actividades alcanzan altos niveles de preocupación y apoyo públicos, inmediatamente después de un gran evento, pero pueden tender a debilitarse a medida que el evento se aleja en el tiempo. En el caso de Chile se ratifica la hipótesis de que la recurrencia de eventos potencialmente causantes de grandes desastres, puede tender a fortalecer la prevención y preparación, reduciendo la vulnerabilidad del sistema en su conjunto. Sin embargo, esta vulnerabilidad puede ser muy variada cuando se presenta un grado muy considerable de heterogeneidad estructural.

Palabras clave: Choques exógenos; vulnerabilidad; prevención; heterogeneidad 


\section{EXOGENOUS THREATS AND VULNERABILITIES: EARTHQUAKES, INTERNATIONAL CRISIS AND DEVELOPMENT IN CHILE, 1930-2010}

Potentially catastrophic natural events (such as the major earthquakes affecting the Chilean territory from time to time) and international economic crises share one characteristic: they are both exogenous events for a small open economy. In both cases, the vulnerability of the socioeconomic system is largely determined by preventive measures and the level of emergency preparedness. These activities reach high levels of public interest and support immediately following a major event, but this tends to weaken as the event recedes in time. For the Chilean case, the hypothesis that recurrence of potentially disastrous events strengthens preventive measures and preparedness, thereby reducing system vulnerability, is confirmed. Nevertheless, vulnerability may vary greatly depending, to a considerable degree, on structural heterogeneity.

Keywords: Exogenous shocks; vulnerability; prevention; structural heterogeneity; Chile. 


\section{AMENAZAS Y VULNERABILIDAD}

Desde una perspectiva general, que considere amenazas naturales puras, "antropogénicas" y mixtas, la vulnerabilidad de un sistema puede definirse como la propensión a sufrir transformaciones significativas como consecuencia de la interacción entre procesos externos o internos ${ }^{1}$. Ello implica que los desastres no son un resultado directo de la amenaza y su magnitud, sino de la vulnerabilidad del sistema.

Además, implica que los desastres no son la única consecuencia posible de un choque ('shock'). También son posibles consecuencias positivas, es decir, cambios profundos (en los sistemas) que abren la posibilidad de desarrollos superiores. Este enfoque "sistémico" tiene algún parentesco con la constatación de que el "ideograma" chino que representa el concepto de crisis (situación en que una entidad o proceso se enfrenta a la posibilidad de cambiar radicalmente o desaparecer) combina los ideogramas que representan peligro y oportunidad.

Las crisis económicas internacionales adoptan, en general, la forma de choques de demanda, que golpean a economías pequeñas, abiertas y excesivamente especializadas $^{2}$ a través de fuertes caídas en los volúmenes exportados y en sus términos de intercambio del comercio exterior. La capacidad productiva no es plenamente utilizada, el desempleo se incrementa radicalmente y la sociedad se desestabiliza económica, social y políticamente.

Los casos más importantes de crisis económicas externas, que adoptaron la forma de choques de demanda, desde el punto de vista de la economía chilena, en los últimos 100 años, fueron la Crisis del Salitre, que se abre a partir de fines de la Primera Guerra Mundial; la Gran Depresión de los años 1930, que golpeó a Chile con mayor fuerza que a ningún otro país de acuerdo a un estudio de la Liga

1 Gallopín (2003), plantea esta visión "sistémica” aplicable no sólo a los sistemas humanos o sociales sino también a los sistemas naturales y socio-ecológicos.

2 Con comercio exterior complementario, es decir con una actividad exportadora muy difícil de redireccionar hacia la producción de sustitutos de las importaciones, ni siquiera a mediano plazo. 
de las Naciones³ la Crisis Asiática, a fines de los 1990 y la Crisis actual, que se inicia en 2008.

Sin embargo, también ha habido choques externos mixtos (de oferta y demanda) en el caso de las economías importadoras netas de ciertos productos cruciales, como en el caso como los choques petroleros estanflacionarios ${ }^{4}$ que se desataron a fines de 1973 y en 1980 y afectaron significativamente a la economía chilena.

\section{Gráfico 1}

\section{Logaritmo natural del PIB per capita en Chile 1910 - 2010}

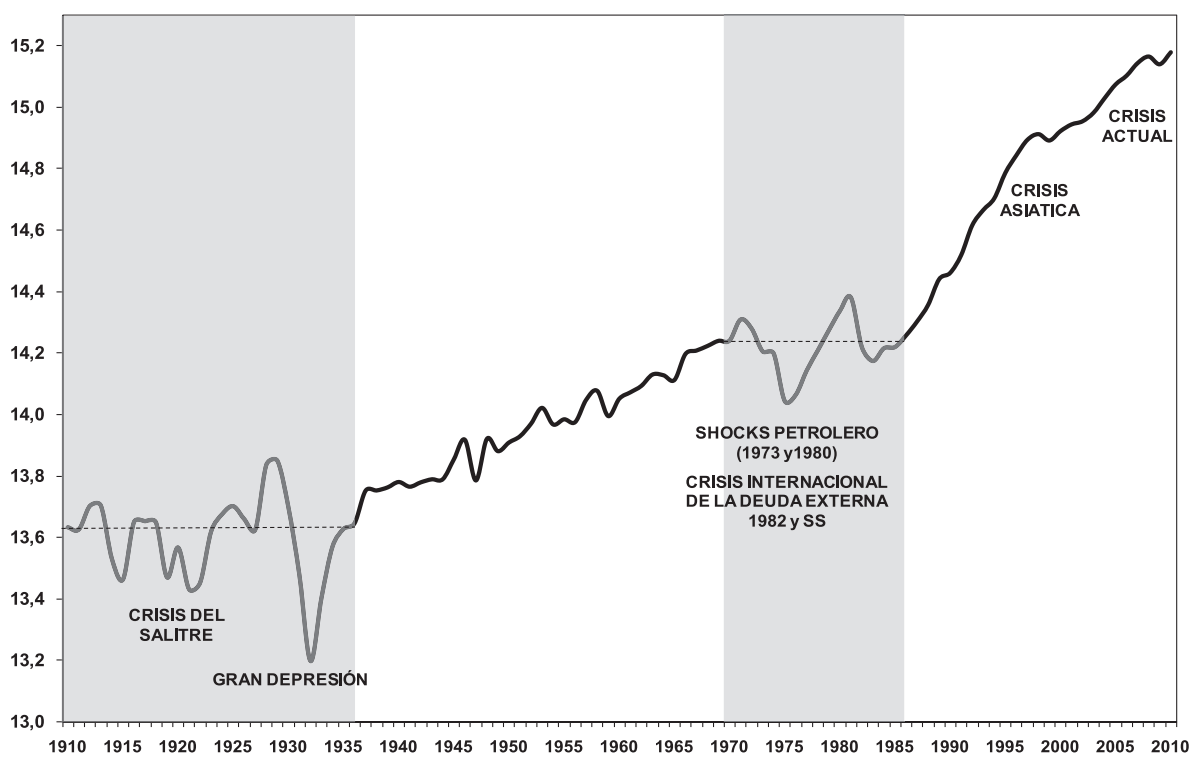

Fuente: Elaboración propia según los datos de: Díaz et al (2007), Banco Central de Chile, INE.

Esos choques estanflacionarios golpearon con gran fuerza a la economía chilena, que era particularmente vulnerable en ambas coyunturas: en el primer caso, por los enormes desajustes económicos, sociales y políticos internos que se desarrollaron a comienzos de los 1970 y, en el segundo caso, por la excesiva desregulación y

3 Un estudio encargado por la Liga de las Naciones identificó a Chile como la economía que sufrió la caída más aguda en la actividad económica interna como resultado de la Gran Depresión; ver Pinto. (1973:168). Esto es ratificado por estudios recientes como, por ejemplo, Maloney (2002:142).

4 En economía, estanflación (stagflation) significa, inflación con alto desempleo, el cual puede ser resultado de un estancamiento o, incluso, una recesión económica. 
la liberalización descontrolada, que amplificó los efectos del choque cuando aquel se propagó internacionalmente en la forma de una crisis internacional de la deuda externa que se desata en 1982 .

En los hechos, el largo período de estancamiento de la economía chilena que abarca desde el Centenario hasta mediados de los años 1930, pasando por la Primera Guerra Mundial, la Crisis del Salitre y la Gran Depresión de los años 1930, período que se caracteriza, además, por la alta conflictividad social e inestabilidad política que refuerzan y propagan los problemas económicos, se repite en un nuevo período de estancamiento prolongado, entre 1970 y 1985, presentándose una interacción y reforzamiento mutuo de los choques económicos externos, la conflictividad social y la inestabilidad política internas.

En cuanto a los terremotos, se trata, sin duda, de choques de oferta, como también lo son las grandes tormentas y otros eventos naturales potencialmente catastróficos.

Sin embargo, también es ampliamente aceptado que una vez superada la emergencia y la rehabilitación, la fase de reconstrucción puede promover altas tasas de inversión y, en consecuencia, un dinamismo económico importante, lo cual depende, por cierto, de la magnitud del choque inicial de oferta y del nivel de utilización de la capacidad productiva previo al evento.

En efecto, una economía que esté utilizando plenamente sus recursos (en pleno empleo) y que reciba un choque de gran magnitud en cuanto a los activos productivos involucrados, debería experimentar problemas de abastecimiento durante la emergencia y la rehabilitación pero ellos podrían traducirse en presiones inflacionarias en la fase de la reconstrucción. Esto último no tendría por qué ocurrir si la situación inicial, anterior al sismo, es caracterizada por una fuerte subutilización de la capacidad productiva, especialmente en el sector de la construcción. Tal fue el caso, en Chile, en el gran terremoto de 1960, antecedido por un ańo recesivo, como también en el gran terremoto de 2010.

A diferencia de las crisis económicas internacionales, los grandes terremotos son fenómenos exógenos que no pueden evitarse ${ }^{5}$, ni en cuanto a su magnitud ni en cuanto a su frecuencia, ni siquiera con una acción concertada a escala global. En

5 Aunque actividad sísmica de magnitud más pequeña puede ser y ha sido provocada por explosiones nucleares subterráneas. 
este aspecto podrían diferenciarse, en la frecuencia y quizás en la magnitud, de otro tipo de amenazas, aparentemente naturales, que podrían atribuirse, por lo menos en parte, al proceso de "calentamiento global", y serían, por lo tanto "antropogénicas", aunque todo esto está abierto a debate.

Los sismos más importantes que habían afectado a Chile en los últimos 100 años son: el terremoto de Chillán, en 1939 (alrededor de 30.000 muertos y varios cientos de millones de dólares de los EEUU en pérdidas ${ }^{6}$ ), el terremoto de Valdivia (alrededor de 6.000 muertos y varios cientos de millones de dólares de los EEUU en pérdidas), en 1960 y, el más reciente, el terremoto del 27 de febrero de 2010, que tuvo epicentro en la Región del Maule y afectó con gran fuerza a varias regiones del país, generó entre 400 y 500 muertos y desaparecidos pero varios miles de millones de dólares en pérdidas.

Los cálculos, en términos de pérdidas económicas, varían considerablemente, en un rango que va desde unos 8.000 a unos 30.000 millones de dólares de los $\mathrm{EEUU}^{6}$, aunque la segunda cifra, precisada por el Gobierno en torno a 29.662 millones de dólares de los EEUU incluye una estimación de los daños indirectos, es decir, de la producción perdida a partir de la emergencia y hasta que se complete la reconstrucción, del orden de 7.600 millones de dólares de los EEUU. Considerando estos antecedentes puede estimarse que la pérdida, en términos de stock de capital productivo, estaría entre unos 8.000 y unos 22.062 millones de dólares de los EEUU, es decir entre un 1,6\% y un 4,4\% del stock de capital productivo estimado a través de la metodología de inventarios perpetuos.

Dado que las cifras oficiales, de agosto $2010^{7}$, estimaron la brecha inicial entre PIB tendencial y PIB efectivo en un 5\% en 2009 y dada la caída en la tasa de inversión ocurrida en 2009 (llegando a un nivel del orden de un 25\% del PIB tendencial, lo cual no permite suponer una expansión de la capacidad produc-

6 Cifras más parecidas al límite inferior fueron planteadas tempranamente por consultores privados, como por ejemplo, Matías Braun, Director de Estategia del Banco de Inversiones IM Trust, que estimó en menos de 8.000 millones de dólares de EEUU las pérdidas totales, incluyendo la infraestructura pública y las pérdidas privadas (La Tercera, 20 de marzo de 2010, pp. 1 y 50), mientras que cifras más cercanas al límite superior son frecuentemente citadas por el Gobierno (El Mercurio, http://diario.elmercurio.cl/detalle/index.asp?id=\{f94c2fcf-f412-4df4$87 \mathrm{f1}-\mathrm{f} 73 \mathrm{ce} 1 \mathrm{a} 26 \mathrm{ad} 3\}$ y son recogidas por la International Disaster Database antes mencionada.

7 Ministerio de Hacienda de Chile (2010), Acta de resultados del Comité Consultivo del PIB tendencial, http://www.dipres.cl/572/articles-70119_doc_pdf.pdf. 
tiva superior a un 5\% en 2010 respecta a 2009), la pérdida de stock de capital causada por el sismo y sus réplicas debería estar cercana al límite inferior del rango mencionado en el párrafo anterior, es decir, más bien lejos de las cifras difundidas por el Gobierno, porque, de lo contrario sería muy difícil explicar los ritmos de crecimiento de 6,5\% y 7\% anual en 12 meses, que se observaron en el segundo y tercer trimestres de 2010, sin presiones inflacionarias significativas.

En otras palabras, el efecto macroeconómico de un terremoto de esta magnitud, en una economía y un país con la vulnerabilidad específica de Chile, no parece ser tan cuantioso comparado con los efectos de grandes choques provocados por crisis económicas internacionales que "destruyen" capital productivo a través de la caída en el ritmo de inversión que se produce a consecuencia de la desconfianza de los inversionistas y la subutilización de la capacidad productiva preexistente. Los terremotos parecen tener un efecto neto expansivo, sobre la producción, a mediano plazo. Eso es, por lo demás, lo que parecen sugerir diversos estudios sobre el tema (ver Albala-Bertrand, 1993). Por ejemplo, Loayza et al. (2009) encuentran que las tormentas y los terremotos tienden a tener un efecto neto positivo sobre el crecimiento, a mediano plazo (en un contexto analítico de pleno empleo), debido a que la reducción del stock de capital por persona empleada aumenta, posteriormente, el ritmo de inversión. Este trabajo desagrega por tipos de países, más o menos desarrollados, tipos de desastres y sectores productivos afectados.

\section{ECONOMIA POLITICA DE LOS GRANDES SISMOS}

Los grandes sismos son amenazas de baja frecuencia y grandes consecuencias potenciales (Alesch y Petak, 1986) y esto le da un sello muy particular a la economía política de la prevención ${ }^{8}$, tanto a aquella que se orienta a mitigar sus potenciales efectos, como a aquella que busca traspasar parte del riesgo (es decir, parte de los daños) a otros agentes (seguros y reaseguros).

8 La prevención tiene que ver con el establecimiento de normas de construcción de edificaciones, infraestructura y redes de servicios básicos y normas sobre uso de suelos, suficientemente exigentes como para reducir a un mínimo aceptable la posibilidad de daño a las personas y para asegurar la operación de la sociedad bajo condiciones de emergencia. Ello incluye el aseguramiento del cumplimiento (enforcement) de tales normas y el aseguramiento de los riesgos residuales para facilitar el financiamiento de su reparación, reconstrucción o reposición. 
También le da un sello particular a la preparación ${ }^{9}$ adecuada para actuar, efectivamente, en la emergencia misma, un sello que estimamos similar al estado de preparación (preparedness) que deben tener las Fuerzas Armadas para movilizarse en el caso de un escalamiento de un conflicto, con la diferencia de que la preparación militar es parte de la disuasión y, por lo tanto, de la prevención, mientras en el caso de las amenazas naturales ello no ocurre.

En el caso de los terremotos, los beneficios asociados a las medidas y gastos preventivos no son apreciados (subjetivamente) como incrementales respecto de la situación anterior al evento, excepto si la población percibe el riesgo (el daño probable) como algo importante. Algo similar ocurre con los beneficios asociados a la preparación para la emergencia.

Todo esto se asemeja, de nuevo, a la forma en que a veces se enfoca el gasto en defensa, sólo que en este caso es posible generar hechos políticos que "refresquen la memoria" de los ciudadanos sobre la importancia de incurrir en tales gastos (dentro de ciertos límites). Además, en el caso de la defensa, existen intereses corporativos que se preocupan de ello. Esto no ocurre, ni de lejos, en el caso de la prevención y preparación para emergencias de origen natural. De manera que, en este caso, el rol de los ciudadanos y la importancia de su preocupación efectiva, es más decisivo.

La recurrencia de eventos sísmicos, aunque sean de menor magnitud o de proximidad relativa (por ejemplo, en otras áreas del mismo país), permite mantener un nivel más alto de preocupación permanente por los aspectos de más difícil aplicación efectiva (enforcement) que los grandes eventos sísmicos (y el desarrollo económico alcanzado al momento que ocurren) permiten introducir en la normativa e institucionalidad preventiva y de respuesta a las emergencias.

Una versión "estilizada" (simulada) del contraste entre preocupación pública ${ }^{10}$ y probabilidad de ocurrencia de grandes eventos sísmicos, en Chile, puede observarse en

9 La preparación (preparedness) tiene que ver con los planes y procedimientos, la organización (incluyendo, especialmente, las comunicaciones), la administración de la ayuda (disponibilidad, financiamiento y logística), el entrenamiento de personal especializado y la educación de la ciudadanía.

10 Aunque no se cuenta con encuestas que permitan hacer un seguimiento a la preocupación sísmica de la población, podría intentarse un estudio de contenido de los medios de comunicación, pero ello no solo trasciende los límites de este ensayo sino que es muy poco probable que 
el Gráfico 2. La preocupación máxima se produce inmediatamente ocurrido un gran evento sísmico (100), pero ella se va reduciendo paulatinamente a los largo de los siguientes 60 meses, es decir, lenta y gradualmente, si se juzga desde el punto de vista político-electoral, pero muy rápidamente si se juzga desde el punto de vista geológico.

\section{Gráfico 2}

Curvas de probabilidad y de preocupacion sobre un gran sismo

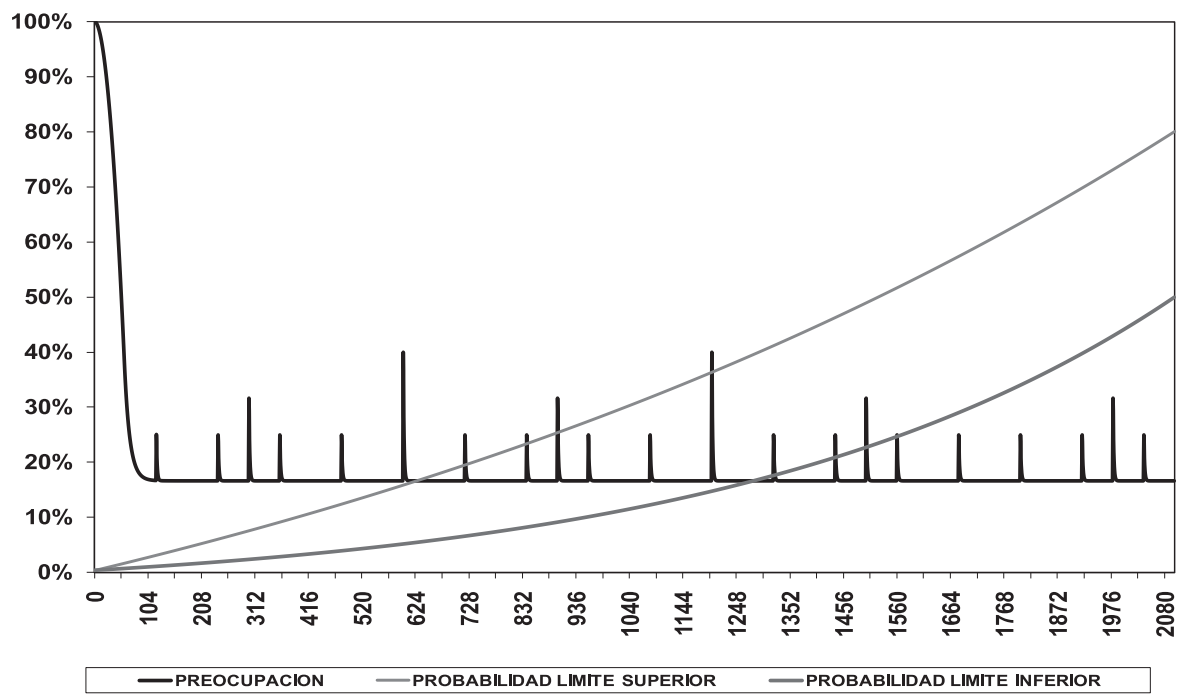

Fuente: Elaboración propia

La amplitud de la banda de probabilidades que hemos incluido en el Gráfico, supone niveles de conocimiento que probablemente no están disponibles ni siquiera entre los expertos, y no pretendemos que sea posible establecer cuál es la distribución de las probabilidades dentro de la banda (aunque los desplazamientos de las placas terrestres y la acumulación de energía que ellos suponen, parecen ser monitoreables, en la actualidad, con mucha mayor exactitud que nunca antes).

En realidad, el comportamiento de la preocupación de la población podría considerarse como el resultado de lo que la abrumadora mayoría percibe como incertidumbre, es decir, el comportamiento propio de personas que deben tomar deci-

pueda ir más allá de la prensa escrita, lo cual implicaría ciertos sesgos en cuanto a los emisores y receptores de tales mensajes. 
siones sin tener la información suficiente como para calcular probabilidades más o menos acotadas para los distintos escenarios relevantes. Wakker (2008) define la incertidumbre como el desconocimiento de las probabilidades de los distintos desenlaces posibles y, por lo tanto, la imposibilidad ("radical" dirían los postkeynesianos) de calcular los riesgos. El concepto postkeynesiano de incertidumbre es "radical". El futuro es imprevisible. Se desconoce, con exactitud suficiente, el conjunto de desenlaces posibles y las probabilidades que deben asignarse a cada uno. En consecuencia, es crucial la confianza de quien decide, lo que implica, en el caso de la economía, que el motor del emprendimiento y la inversión proviene de los 'animal spirits'.

De manera que, inmediatamente después de un gran evento sísmico, la preocupación no cae inmediatamente (no acompaña a la probabilidad de que el gran evento se repita). Ello genera un período de oportunidades políticas, que permite introducir reformas en la normativa y la institucionalidad muy relevantes, correspondientes al nivel de desarrollo económico y social alcanzado por el país con su crecimiento a lo largo de los años. También podría permitir plantearse iniciativas de carácter más amplio, relacionadas con el impulso del proceso de desarrollo en su conjunto.

Con el tiempo, la banda de probabilidad de ocurrencia del evento se incrementa pero la preocupación se mantiene a niveles relativamente bajos. En otras palabras, aunque no disponemos de registros de opinión pública sobre el tema, la experiencia parece indicarnos que la incertidumbre se traduce en bajos niveles de preocupación (tal vez injustificadamente bajos) en las décadas posteriores a un gran evento sísmico, e injustificadamente altos niveles de preocupación (por la posibilidad de que se repita el evento) en el período inmediatamente posterior a un gran sismo.

La magnitud del mega choque, la frecuencia de los choques intermedios y el 'piso' de preocupación persistente, determinan la continuidad del esfuerzo preventivo efectivo que no es homogéneo en cuanto a los distintos sectores sociales y que es más efectivo en el cumplimiento de las normas constructivas que en lo que se 
refiere al cumplimiento de normas estrictas de ordenamiento (reordenamiento) territorial ${ }^{11}$.

Este comportamiento parece tener cierta similitud con la forma en que los actores económicos reaccionan frente a una crisis económica (especialmente una crisis financiera) ya desatada (altos niveles de preocupación, cautela excesiva y desconfianza generalizada) que con el tiempo (con el paso de algunos años y más aún cuando pasan varias décadas) le abre paso a un período de bajos niveles de preocupación, incluso llegando a permitir que se repitan conductas que aumentan la vulnerabilidad del sistema debido a la existencia de niveles de preocupación injustificadamente bajos. Esto último parece ser parte de la lógica de los grandes ciclos de la economía de mercado capitalista.

En Chile son bastante frecuentes sismos sobre el Grado 7 en la escala de Richter, pero grandes sismos sobre el Grado 8 son más espaciados y sobre el Grado 8,5 son mucho menos frecuentes: su repetición en una misma área del territorio parece tomar muchas décadas (más de un siglo y tres cuartos en el caso del último terremoto). Estos son períodos geológicamente cortos pero política y electoralmente muy largos.

\section{PROPENSIÓN SÍSMICA, PREVENCIÓN Y PREPARACIÓN}

Estudios internacionales recientes demuestran que la mortalidad es menor en países con una mayor propensión a experimentar terremotos, debido a que, comparando con otras alternativas para el uso de los recursos, el rendimiento de la prevención es mayor (ver Keefer et al., 2010). Estos estudios controlan el efecto de la magnitud y localización de los terremotos a diferencia de lo planteado por Khan (2005).

11 Piénsese en el costo de hacer efectiva la prohibición de construir viviendas en zonas de riesgo, tema que, de acuerdo a la experta norteamericana Laura Kong (2010) Directora del Centro Internacional de Información de Tsunamis (CIIT) desde 2001, no se ha logrado resolver de manera plenamente satisfactoria ni siquiera en los países más desarrollados. 
Aunque la destrucción de construcciones y equipos es mayor en los países más desarrollados, debido a la intensidad de capital de los mismos, la mortalidad es mayor en los países más pobres (Kenny, 2009:3).

Ahora bien, en los países más pobres la prevención sísmica es menor porque provee rendimientos que son más bajos o más inciertos (en cuanto a su materialización) que usos alternativos de los escasos recursos disponibles, con efectos más inmediatos y cotidianos en el bienestar de la población. En los países más pobres la mayoría de la gente no muere en desastres específicos y ello puede conducir a que se considere más barato y más fácil prevenir otras causas de muerte. Esto puede agravarse cuando la pobreza se combina con gobiernos con menos incentivos para proveer bienes públicos. Tal sería el caso de las democracias jóvenes, poco desarrolladas, los gobiernos autocráticos con partidos gobernantes menos institucionalizados y los regímenes con altos niveles de corrupción (según Keefer et al, 2010:2-3).

Obviamente, normas más exigentes y un cumplimiento más estricto de ellas, implican costos económicos, administrativos y políticos más altos pero reducen la magnitud de los efectos y, por lo tanto, debería reducir también los requerimientos sobre las empresas aseguradoras, de manera que deberían reducir las primas e incrementar el acceso a este aspecto de la prevención.

Normas más exigentes y un cumplimiento más estricto de ellas, también deberían permitir una mejor respuesta frente a la emergencia, porque desde el punto de vista logístico y productivo no es lo mismo evitar daños que financiar la reconstrucción y reposición de las instalaciones y equipos perdidos.

Ese debería ser el criterio especialmente en lo que se refiere a infraestructura y servicios básicos, ya que los planes y procedimientos de respuesta ante una emergencia dependen, en su eficacia (que incluye la rapidez con que se despliegan), del nivel de destrucción de la infraestructura básica generado por el sismo, especialmente en el caso de las vías, las redes de energía, las comunicaciones, el agua potable y el alcantarillado y las edificaciones que albergan centros fundamentales como los centros de mando, los centros de comunicaciones, los hospitales, los establecimientos educacionales, etc. 
También dependen de la capacidad de diagnóstico, evaluación y toma de decisiones de las autoridades, porque las medidas de emergencia, adoptadas oportunamente, evitan "réplicas sociales" que pueden agravar la situación, como el desarrollo de situaciones en que se pierde el control de la población y ocurren daños a la propiedad pública, privada y a las personas.

\section{HETEROGENEIDAD PRODUCTIVA, DESIGUALDAD Y VULNERABILIDAD}

Los desastres nunca son "naturales": Las amenazas pueden ser "naturales" o "antropogénicas", pero los desastres, es decir la ocurrencia de daños masivos que pueden generar efectos negativos persistentes, se producen cuando una amenaza se hace efectiva y golpea a una población vulnerable.

Es esa vulnerabilidad la que debe reducirse con medidas preventivas, pero no cabe duda que la heterogeneidad productiva y la desigualdad social generan situaciones de vulnerabilidad focalizada en los sectores más pobres. Como se sabe, ello no significa que todos los más pobres sean vulnerables a un evento violento de alto potencial destructivo y tampoco significa que los únicos vulnerables sean los pobres, pero no hay duda de que la abrumadora mayoría lo son. De hecho, un desastre mal gestionado puede acentuar la vulnerabilidad de ciertos sectores y generar lo que se ha llamado un "círculo de la vulnerabilidad" (BID, 2007:9) que no es otra cosa, a nuestro entender, que un "círculo de la pobreza".

El cumplimiento de las normas y el financiamiento adecuado de las entidades fiscalizadoras pueden tender a debilitarse con el tiempo y se tornan menos efectivos, aun en los sectores de menores ingresos en los que predomina la ocupación de terrenos riesgosos, la autoconstrucción y las ampliaciones artesanales ${ }^{12}$,

12 Incluso validadas, hasta cierto punto, por las autoridades, con prácticas como las llamadas leyes del 'mono' (dibujo o plano sencillo), leyes especiales aprobadas y promulgadas para facilitar la regularización de construcciones en sectores de menos ingresos: Ley 19.583 del 14 de septiembre de 1998, prorrogada por la Ley 19.797 y replanteada en la Ley 20.251 del 4 de marzo de 2008 -esta última con vigencia de tres años pero con seis años en el caso de viviendas erigidas en 'zonas de catástrofe'. 
y en sectores 'aspiracionales' 13 , enfrentados a fuertes restricciones presupuestarias y a empresas inmobiliarias-constructoras sin reputación (que entran y salen del mercado) y, también, en sectores de bajos ingresos, "beneficiarios" de viviendas sociales mal fiscalizadas.

El resultado es que la vulnerabilidad puede crecer de manera inversa a los niveles de ingreso de los hogares porque resulta económica y políticamente poco rentable asumir el costo de la aplicación estricta de las normas en estos sectores, sobre todo cuando los ciclos político-electorales son tan cortos.

En definitiva, puede acumularse una vulnerabilidad que se traduciría en riesgos (daños potenciales) que en muchos casos tampoco están asegurados, ni siquiera parcialmente, de manera que el seguro implícito es la capacidad que el Estado tenga de subsidiar a estos sectores una vez que el desastre se genere.

En el caso chileno es evidente que la aplicación de normas sobre construcción de viviendas es mucho menos clara y mucho menos efectiva en el caso de las viviendas producidas con técnicas tradicionales (en muchos casos utilizadas, también, como edificaciones de uso múltiple, que incluyen actividades productivas), con un fuerte componente de autoconstrucción. Algo similar ocurre con las ampliaciones informales que ocurren a partir de construcciones estandarizadas y, ciertamente, con el cumplimiento de las normas de ordenamiento territorial. En consecuencia, la vulnerabilidad está fuertemente focalizada en los sectores con menos recursos. El cumplimiento efectivo (enforcement) de las normas vigentes o la introducción de normas en aquellos casos en que no existían, es tremendamente costoso en términos económicos y en términos políticos ${ }^{14}$.

Aun si funcionara perfectamente el mercado de prevención de riesgos sísmicos, el caso es que las normas más exigentes han ido siendo introducidas a lo largo de un proceso histórico, especialmente después de los terremotos de 1939 y 1960, pero el mercado de edificaciones nuevas es sólo una parte del stock total de edificaciones y de ellas un porcentaje importante son edificaciones informales o edificaciones de mala calidad, que tampoco están a la altura de las normas establecidas.

13 Sectores pertenecientes a estratos medios bajos en ascenso, con tendencia de llevar su consumo hasta los límites de su capacidad presupuestaria o, incluso, más allá.

14 Puede decirse, en este marco, que es muy probable que el déficit habitacional haya estado subestimado a lo largo de muchas décadas, porque no se ha calculado con base en criterios de resistencia a grandes sismos o localización segura. 
La informalidad, más o menos regularizada ${ }^{15}$, tiene una alta incidencia en sectores de bajos ingresos. Las construcciones antiguas no han sido tratadas preventivamente, ni en el caso de los monumentos nacionales ni en el caso de las viviendas y edificaciones menos sobresalientes que caracterizan a zonas rurales muy importantes que fueron particularmente golpeadas por este sismo. El 27 de febrero Chile experimentó un terremoto cuyo balance es un promedio entre aquellas áreas del país que están cerca del mundo desarrollado, aquellas otras que están más atrasadas y aquellas, también existentes, aunque menos importantes como porcentaje de la población total, que permanecen ancladas en la subsistencia y en tecnologías completamente anacrónicas.

La prevención implica resistencias y oposiciones por parte de sectores de la industria de la construcción, propietarios de terrenos, pobladores y todo tipo de sectores que no le asignan una probabilidad y un riesgo suficientemente altos a los efectos de la catástrofe, o que esperan tener la suerte de librarse de las consecuencias y obtener la ayuda complementaria que necesitarán en caso de que ella ocurra. Tomó 38 años, entre el terremoto de 1933 y la ordenanza, pionera, de 1971, para que Long Beach estableciera políticas de reducción de los riesgos sísmicos en edificios no reforzados de albañilería que quedaron en pie en aquel terremoto. Fue un proceso extremadamente difícil. En Los Angeles tomó 48 años establecer una ordenanza similar (Alesch y Petak, 1986: 179.).

Los grandes terremotos crean una oportunidad para generar normas más estrictas y mecanismos de aplicación efectiva (enforcement) más eficaces, pero hay que aprovechar el momento y evitar que las urgencias de la "reconstrucción" y su carácter masivo, generen una nueva fuente de incumplimiento estricto de las normas. En tiempos normales es muy difícil que las autoridades prioricen iniciativas preventivas y remediales que tienen un bajo efecto positivo demostrable y un alto costo de oportunidad y resistencias, como, por ejemplo, erradicar asentamientos urbanos de zonas de riesgo o dedicar recursos a la inspección y mantenimiento de edificaciones ya existentes, que han sido desarrolladas con técnicas constructivas que no cumplen con las normas o que se deterioran por el paso del tiempo. Es bien sabido, por ejemplo, que el concreto requiere monitoreo y mantenimiento, lo cual no está ampliamente incorporado al conocimiento de la ciudadanía.

15 A través de leyes especiales ya mencionadas, conocidas popularmente como leyes "del mono". 


\section{PREVENCION DE CRISIS ECONÓMICAS VS. PREVENCIÓN DE DESASTRES NATURALES}

Las grandes crisis económicas internacionales, al igual que los grandes terremotos, son fenómenos que no son frecuentes (no confundir con las fluctuaciones, menos abruptas, que son típicas de la actividad económica), tienen un potencial de daño enorme y son exógenos desde el punto de vista de una economía pequeña, es decir, no pueden evitarse, desde el punto de vista de ella.

Ello implica que una economía pequeña estará mejor resguardada si se adoptan medidas preventivas que amortigüen los efectos de la crisis y si se cuenta con un nivel de preparación y financiamiento adecuado para desplegar las medidas de emergencia transitoria que se requieran, para atenuar aún más los efectos. En otro nivel, las medidas preventivas deberían incluir la participación, decidida, en los esfuerzos internacionales orientados a eliminar, reducir y/o regular la presencia de factores de amplificación de fluctuaciones que son recurrentes, incluyendo planes de emergencia para contener elementos desestabilizadores.

A partir de la experiencia generada por crisis anteriores el país desarrolló una institucionalidad, normativa y prácticas tendientes a manejar shocks macroeconómicos, pero no avanzó en la misma medida en la prevención basada en una diversificación productiva mayor, una reducción de la heterogeneidad productiva extrema (coexistencia de tecnologías de punta con tecnologías anacrónicas) y una agregación de valor más intensa. En otras palabras, se avanzó menos en los aspectos más reales que financieros de la "prevención". De todas maneras, la diversificación de productos exportados, exportadores y, sobre todo, mercados de destino, probó ser importante en la crisis más reciente.

En el origen del algoritmo fiscal que permitió generar Fondos para enfrentar pasivos futuros (fundamentalmente previsionales) y suavizar las fluctuaciones económicas, no estaba la idea de proveer una cobertura contra crisis internacionales de gran magnitud como la que se desata en 2008. Sin embargo, el desendeudamiento neto del Estado, que ello generó, y el hecho de que la crisis internacional fuera tan asimétrica como para que el dinamismo de ciertos países, como China, permitiera disfrutar, a Chile, de altos precios del cobre y muy buenos términos de 
intercambio, amortiguaron las tradicionales consecuencias de una crisis externa, evitando que se presentaran presiones tendientes a depreciar la moneda nacional.

Sin embargo, al igual que en lo que se refiere al sesgo financiero de la estrategia preventiva quedan en pie ciertas preguntas: ¿Se justificaba ahorrar en comunicaciones ${ }^{16}$, vialidad, transporte, hospitales y establecimientos carcelarios hasta el punto de que instalaciones importantes colapsaran al ocurrir un sismo como el del 27 de febrero? ¿Era necesario tener fondos tan cuantiosos ahorrados en vez de inversiones reales preventivas? ¿Se justificaba tener una institucionalidad de emergencia tan escuálida?

Cada vez resulta más evidente que se postergaron modernizaciones y gastos fundamentales que pudieron haber permitido una respuesta más adecuada frente a la emergencia ${ }^{17}$. La reorganización total de la institucionalidad que debe enfrentar las emergencias, anunciada por el actual Gobierno, ilustra claramente este punto ${ }^{18}$.

\section{TRASPASANDO EL RIESGO}

La prevención que busca evitar completamente los efectos de una amenaza (prevención "absoluta" o de "tolerancia cero"), implica un cierto nivel de sobredimensionamiento, mientras que los seguros (que buscan compensar los efectos, una vez producidos) implican una estimación de los daños probables que, como nunca son generalizados, ni siquiera en construcciones con las mismas características, implican costos probablemente menores que el nivel de prevención "absoluta" antes mencionado.

En este sentido, normas demasiado exigentes acompañadas de mecanismos de aseguramiento de su cumplimiento efectivo, pueden tener un costo económico,

16 El comportamiento de las redes es analizado por Barros (2010).

17 Esto es, lamentablemente, muy evidente en el caso de las víctimas de los maremotos posteriores al terremoto del 27 de febrero, los cuales cobraron un importante número de vidas.

18 Ver, por ejemplo, "La ONEMI y el SHOA están en proceso de reestructuración, pero aún están lejos del ideal”, El Mercurio, 27 de agosto de 2010. También las declaraciones del Subsecretario del Interior, Rodrigo Ubilla: "El énfasis del Gobierno es proponer al Congreso una legislación que reordene y proponga una nueva institucionalidad para enfrentar las emergencias y la protección civil", 3 de noviembre 2010, http://informa.gobiernodechile.cl/comunicados-archivo/ 
político y administrativo superior a los beneficios, de manera que ciertos aspectos de los dańos probables (que no pongan en riesgo la integridad física de las personas) serían siempre abordados de manera más "económica" recurriendo a los seguros.

La sociedad debería establecer con bastante claridad cuál es el nivel de daños que está dispuesta a tolerar y a reparar con posterioridad. Ello debería implicar asumir, también, de manera cierta, el costo de las primas de los seguros correspondientes y el costo, en términos de ahorro (no invertido en activos sujetos al mismo riesgo) necesario para asumir los "deducibles" y el riesgo residual (no transferido). Estos seguros pueden ser privados o pueden ser públicos y lo más razonable es que sean mixtos.

Ello implica la necesidad de que existan seguros privados adecuados, tal vez obligatorios en ciertos casos, como aquellas construcciones en que los dańos pueden afectar la habitabilidad de inmuebles vecinos mientras no se repare o se demuela el inmueble siniestrado. También es razonable que exista un Fondo Nacional que permita apoyar las medidas preventivas en materia de edificaciones de los sectores más pobres y la reconstrucción o reparaciones una vez ocurridos los daños. Es necesario pensar con cuidado la combinación público-privada. El terrorismo, por ejemplo, ha demostrado que cuando el Estado asume los riesgos que el mercado de seguros no es capaz de proveer, ello puede reducir, adicionalmente, el uso de los seguros privados como alternativa. Nell, M. y Richter, A. (2004: 19).

En el caso del mercado hipotecario, que utiliza normalmente seguros con cobertura sísmica, no debería bastar con "salvar vidas y no estructuras", porque estas últimas son las garantías de sus créditos. Estaríamos frente a un efecto humanitario a partir del interés material estricto de los actores del mercado. Sólo la destrucción de aspectos no estructurales menores deberían ser tolerables para los beneficiarios de las garantías hipotecarias, es decir, para los Bancos.

Sin embargo, la crisis hipotecaria que desató la crisis financiera en los EEUU, en 2008, nos enseña que las garantías hipotecarias pueden perder importancia cuando el riesgo de los créditos otorgados se traslada a otros actores o se traslada de manera difusa, a través de innovaciones financieras, de forma que la disciplina propia del otorgamiento de créditos se debilita. Por cierto, los costos adicionales que implican los estudios, diseños y construcciones más exigentes y/o primas de los seguros contra riesgos sísmicos, son traspasados, en buena medida, a los 
compradores de las nuevas edificaciones, lo que encarece los dividendos en los créditos hipotecarios.

De manera que las empresas inmobiliarias, sometidas a niveles considerables de competencia (por lo menos en las ciudades más grandes) o a limitaciones de costos en ciertos estratos medios bajos del mercado, deberían tratar de reducir todos los costos, incluyendo las primas de los seguros.

Es fundamental asegurar condiciones que permitan que los actores de este mercado equilibren la tendencia a la reducción en costos con la necesidad de financiar hipotecariamente (y con seguros) a los compradores, de manera que los seguros tengan un costo que refleje de manera adecuada los grados de cumplimiento de las normas obligatorias y voluntarias que pudieran utilizarse para certificar las construcciones.

De ser así, el resultado neto de estas fuerzas debería ser una construcción que cumpla con normas exigentes y sea, al mismo tiempo, tan barata como sea posible.

De todas maneras, en el mercado de edificaciones nuevas correspondientes a estratos medios y bajos, que opera minimizando costos en todos los frentes, un porcentaje ínfimo colapsó con efectos mortales en el terremoto del 27 de febrero, y un porcentaje muy pequeño, pero un poco mayor, es irrecuperable. Sin embargo, resultó sorpresivo observar daños importantes en edificaciones corporativas en sectores tan importantes como la Ciudad Empresarial, en Santiago, para no mencionar casos similares en las zonas centrales de ciudades importantes más cercanas al epicentro, como Concepción.

\section{FALLAS DE MERCADO Y FALLAS DE ESTADO: PROBLEMAS DE COORDINACIÓN Y CONCENTRACIÓN DEL PODER ECONÓMICO}

La teoría de los mercados "incompletos" se fundamenta en el reconocimiento de que para coordinar sus actividades y compartir los riesgos los agentes económicos deberían realizar transacciones que establecieran sus compras y ventas futuras o les permitieran cubrirse contra los riesgos que surgen de los distintos escenarios posibles (mercados completos), pero ello no ocurre prácticamente nunca (Stiglitz, 2009: 293 y Greenwald et al. 1986). 
Esta es una de las principales razones por las que es necesario generar formas de producción y difusión de información relevante y espacios público-privados de discusión e intercambio de visiones estratégicas, tanto a nivel nacional como a nivel de regiones o ciudades. La cuestión de la reconstrucción plantea el mismo tema, sobre todo en presencia de cadenas productivas destruidas y mallas de proveedores de servicios que han debido cesar sus actividades y dependen de otros, que también han cesado las suyas para reiniciarlas.

El problema es que la recuperación-reconstrucción, se demorará más y será menos inclusiva en ausencia de un Estado capaz de proveer los espacios público-privados de intercambio estratégico que sirvan de marco para la canalización de recursos suficientes como para asegurar el acceso al financiamiento de aquellos que, en condiciones normales, tendrían garantías para responder por los créditos, pero en la situación actual, no pueden demostrar que la recuperación-reconstrucción les proveerá los clientes que perdieron.

Dejados a las fuerzas del libre mercado "incompletas" o, si se prefiere, "miopes", se derrumban los precios de los terrenos urbanos que proveían la base de construcciones y sistemas funcionales que tomó muchos años desarrollar. Los grandes empresarios, más diversificados y con mayor capital social, no enfrentan estas restricciones financieras. Por el contrario, en el marco de la crisis internacional, las tasas de interés son menores que en épocas normales y ellos tienen acceso a ese tipo de financiamiento.

En consecuencia, la "libertad del mercado" puede traducirse en un proceso de concentración aguda de la propiedad del suelo urbano en áreas en que muchas viviendas, viviendas-talleres, viviendas-hospedajes y viviendas-tiendas han sido demolidas y sus propietarios no tienen recursos para reconstruir y reiniciar sus actividades productivas si sus vecinos y clientes tampoco los tienen.

Sabemos que en la economía chilena, la riqueza que se produce en diversas regiones genera ingresos importantísimos que se "filtran" hacia el "exterior", hacia la capital del país y hacia el resto del mundo. Ello debilita las posibilidades del desarrollo regional, porque impide el crecimiento de sectores que enriquezcan el sistema productivo como un todo. Es bien sabido que los que compiten son los sistemas productivos y no las actividades o establecimientos específicos. Y también es bien sabido que uno de los problemas ("fallas") que presentan los 
mercados, en lo que se refiere al desarrollo, es que son "incompletos" es decir, que no existen suficientes mercados en los que se negocien y contraten derechos y obligaciones sobre actividades productivas futuras.

En muchos casos, las construcciones demolidas combinaban funciones residenciales y productivas, a veces en la misma edificación y en otras en edificaciones aledañas. Pero a la descapitalización causada por la destrucción y posterior demolición, se agrega la descapitalización adicional causada por la imposibilidad de "resistir" financieramente las urgencias. Aún cuando sepan que les conviene esperar a que los precios se recuperen, como siempre lo hacen, a mediano y largo plazo, se necesitan recursos para reconstruir y trabajar.

El desarrollo de las zonas más afectadas por el sismo supone su reconstrucción, pero no se agota en ella y es una condición fundamental para hacer posible la renovación del impulso productivo general. No será posible el desarrollo productivo más competitivo, diversificado y sistémicamente fortalecido, que todas las experiencias exitosas nos indican como camino, si se permite que la "masa crítica" de actividades urbanas golpeadas por el desastre, se deslice por una pendiente de descapitalización paralizante.

Es fundamental que el Estado genere "fondos de rescate urbano", capaces de detener esta espiral y coordinar una reconstrucción-recuperación que le ofrezca oportunidades a quienes proveían tantos empleos y servicios fundamentales.

Estamos hablando de fondos que valoricen los terrenos y estructuras que existen al precio que tendrán cuando las ciudades más afectadas se recuperen, fondos que reconozcan que los precios actuales no representan los precios de largo plazo y que el Estado, no los privados, puede asumir esa realidad proveyendo créditos (complementarios al financiamiento privado) contra los bienes raíces existentes pero valorados a precios de futuro. Ese capital permitirá recuperar las actividades y parte importante de las construcciones perdidas y si se enmarca en un plan regulador y una visión urbana de futuro, puede llevarnos mucho más allá que una mera reconstrucción.

Por cierto, el Estado puede proveer los recursos complementarios requeridos contra la garantía que no utilizarán los privados, es decir, contra la plusvalía urbana que el propio desarrollo (reconstrucción-recuperación) generará, de manera que no tiene 
por qué "perder plata" o consumir los activos que destine a estos efectos (en realidad puede hacerlo parcialmente si desea incluir elementos de "subsidio" acotados).

\section{CRECIMIENTO, DESARROLLO Y GRANDES TERREMOTOS}

En el caso de Chile, manteniendo el contrapunto entre "choques naturales" y "choques económicos" podemos observar (en el Cuadro 1), que la Gran Depresión de los años 1930 tuvo un efecto prolongado sobre el Producto Interno Bruto (PIB) per capita y los niveles de 1928-29 no se volvieron a alcanzar hasta terminada la segunda Guerra Mundial en 1945.

En cambio, la Crisis Asiática, de menores dimensiones, se recuperó en dos años y la Crisis actual, que comenzó en 2008 y afectó negativamente la producción en 2009, se superaría durante el presente año, a pesar de su mayor envergadura. Ello se debe a la adopción de medidas preventivas, que se discuten más adelante, y a un escenario internacional más globalizado y más complejo (en cuanto a sus dinamismos) que el de la Gran Depresión.

En el caso de los grandes terremotos, el de Chillán en 1939, aparentemente fue contrarrestado por las fuerzas de la reconstrucción y por el plan de industrialización que comenzó a desarrollar la CORFO, creada por el Presidente Pedro Aguirre Cerda tres meses después del sismo, de manera que se mantuvo cierta inercia de bajo crecimiento, que venía desde antes, hasta fines de la Segunda Guerra Mundial, pero a partir de allí se despliega un dinamismo importante que posteriormente se tornaría espasmódico e inestable. 


\section{Cuadro 1}

Chile: Producto Interno Bruto per capita a precios constantes índices, Año anterior al evento $=100$

\begin{tabular}{|c|c|c|c|c|c|}
\hline \multicolumn{2}{|c|}{ Gran Depresion } & \multicolumn{2}{c|}{ Terremoto De Chillan } & \multicolumn{2}{c|}{ Terremoto de Valdivia } \\
\hline 1928 & 99 & 1937 & 100 & 1958 & 109 \\
\hline 1929 & 100 & 1938 & 100 & 1959 & 100 \\
\hline 1930 & 86 & 1939 & 101 & 1960 & 106 \\
\hline 1931 & 69 & 1940 & 103 & 1961 & 108 \\
\hline 1932 & 52 & 1941 & 101 & 1962 & 110 \\
\hline 1933 & 64 & 1942 & 103 & 1963 & 114 \\
\hline 1934 & 76 & 1943 & 104 & 1964 & 114 \\
\hline 1935 & 80 & 1944 & 104 & 1965 & 112 \\
\hline 1936 & 82 & 1945 & 111 & 1966 & 122 \\
\hline 1937 & 91 & 1946 & 118 & 1967 & 124 \\
\hline
\end{tabular}

\section{$1^{\text {er }}$ SHOCK PETROLERO Y
GOLPE MILITAR}

20 SHOCK PETROLERO Y CRISIS DE LA DEUDA EXT.

\section{Crisis asiatica}

\begin{tabular}{|c|c|c|c|c|c|}
\hline 1971 & 103 & 1978 & 94 & 1996 & 95 \\
\hline 1972 & 100 & 1979 & 100 & 1997 & 100 \\
\hline 1973 & 93 & 1980 & 106 & 1998 & 102 \\
\hline 1974 & 92 & 1981 & 111 & 1999 & 100 \\
\hline 1975 & 79 & 1982 & 95 & 2000 & 103 \\
\hline 1976 & 81 & 1983 & 90 & 2001 & 105 \\
\hline 1977 & 87 & 1984 & 94 & 2002 & 106 \\
\hline 1978 & 93 & 1985 & 95 & 2003 & 109 \\
\hline 1979 & 99 & 1986 & 98 & 2004 & 115 \\
\hline 1980 & 106 & 1987 & 103 & 2005 & 120 \\
\hline
\end{tabular}

Fuente: Elaboración propia partir de Díaz et al (2007) 
El Terremoto de Valdivia encontró a la economía chilena en medio de una recesión provocada por el programa económico del nuevo gobierno del Presidente Jorge Alessandri, que introdujo una reforma monetaria (el "Escudo". Eo), decretó la convertibilidad a la par con el dólar de los EEUU y abrió las importaciones, todo lo cual se tradujo en un impacto negativo sobre los sectores que sustituían importaciones y un incremento considerable de los déficits en Balanza de Pagos.

El Terremoto de Valdivia, a pesar de la destrucción de capacidad productiva ("shock de oferta") no generó una caída en la producción per cápita. Los programas de fomento del sector Vivienda, que se habían introducido antes del terremoto, permitieron sostener un dinamismo que finalmente sucumbió ante la escasez de divisas que se generó hacia fines de 1962. Este penúltimo ensayo con un tipo de cambio fijo, en Chile, desembocó en la devaluación del "escudo", la intensificación de la inestabilidad económica (inflación galopante), social y política, tal como había ocurrido con el patrón oro en 1930 y como volvería a ocurrir en 1982.

Los "shocks" petroleros de 1973 y 1980 fueron tan solo ingredientes adicionales ("las gotas que rebalsaron el vaso") en el marco de coyunturas cuyas tensiones se explican por la crisis, el Golpe Militar y el cambio drástico en las políticas públicas y en la institucionalidad que se produce en 1973 y, en el segundo caso, por la Crisis internacional de la Deuda Externa que es detonada en 1982 por la cesación de pagos de Polonia seguida corto tiempo después por Méjico. Chile, que estaba de nuevo ensayando un tipo de cambio fijo con gran apertura externa (tal como en 1930 y 1960), terminó enfrentando una aguda crisis financiera que obligó a rescatar al sistema bancario y ajustar las políticas económicas para enfrentar el problema de la deuda externa.

De hecho, el terremoto de 1985 se da hacia el final del período de recuperación, luego del cual vendría lo que se ha dado en llamar el "período de oro del crecimiento económico" entre 1986 y 1998, en el cual se duplicó el PIB per capita del país (Gallego y Loayza, 2002), la economía chilena fue una de las cuatro que más crecieron en el mundo y los ritmos que se alcanzaron fueron sobresalientes: 7,2\% promedio anual en términos de Producto Interno Bruto (PIB) y 5,5\% promedio anual, en términos de PIB per capita. 
La Crisis Asiática que golpea al país desde fines de 1998 y especialmente en 1999, tuvo un efecto limitado sobre la producción interna pero marca un punto de inflexión que deja atrás el "período de oro", 1986-1998, sin que se haya recuperado el ritmo de crecimiento desde entonces. Si ese desempeño se hubiera mantenido hasta el presente, Chile sería, hoy, una economía que tendría una producción por habitante en el rango que va desde Corea del Sur e Israel hasta España e Italia, pero ello implicaba completar dos décadas y media de alto crecimiento económico, algo que habría ubicado el caso chileno entre los "milagros económicos" que pueden encontrarse en la historia económica mundial.

Por cierto, la teoría económica del crecimiento establece que los países más desarrollados crecen al ritmo per capita que les permite la innovación productiva, mientras los países "en desarrollo", en la medida que se integren plenamente a los circuitos internacionales de capital y adopten las medidas necesarias para establecer instituciones y políticas públicas adecuadas, deberían crecer más rápidamente, convergiendo hacia los niveles de desarrollo (más altos) y ritmos de crecimiento (más bajos) de los países más desarrollados.

Ello podría explicar una tendencia a la disminución en los ritmos de crecimiento de una economía que ha atravesado por un período de alto crecimiento, pero ello sólo debería ocurrir una vez que tal economía se ha acercado significativamente a los niveles de productividad de los países más desarrollados. Este no es el caso de Chile.

La teoría económica del crecimiento también establece que los países que no han adoptado las medidas necesarias para contar con la institucionalidad y las políticas adecuadas, no convergerán hacia el nivel de los países más desarrollados sino que tenderán a converger hacia niveles menores, de manera que se desacelerarán sus procesos de expansión mucho antes de que alcancen los niveles de productividad requeridos para alcanzar el desarrollo ${ }^{19}$.

Existe la posibilidad de que el ritmo de crecimiento más bajo, que se observa desde el impacto de la Crisis Asiática en 1998-1999, se relacione con la presencia de debilidades muy importantes que no se han abordado con la fuerza y oportunidad requeridas, tal vez porque la fase de expansión acelerada, lograda

19 "Necesarios pero no suficientes", porque el desarrollo implica un conjunto complejo de dimensiones. 
en base a recursos y ventajas ya existentes, postergó la iniciativas necesarias para crear nuevas ventajas (ver, por ejemplo, el análisis de Landerretche, Lanzarotti y Ominami, 2004).

Diversos análisis críticos han subrayado las dificultades que el país ha tenido para plantearse una estrategia de desarrollo más definida, institucionalizar el diálogo social sobre este tipo de temas, convocar esfuerzos públicos y privados, productivos y educativos, orientando y coordinando las iniciativas, para superar las "fallas de mercado" que impiden romper inercias y crear nuevas ventajas.

En el extremo opuesto, el énfasis se pone en las "fallas de Estado" y la orientación sigue siendo privatizar y liberalizar todo lo que se pueda y circunscribir la actividad del Estado a lo indispensable. Existe, por supuesto, un amplio espacio intermedio en el que pueden lograrse compromisos que permitan romper las inercias.

El "empate político" institucionalizado, que parece ser el resultado inevitable del actual sistema político chileno, ha permitido desdibujar, desde el punto de vista del electorado, las diferencias profundas de enfoque que existen sobre diversos tópicos, incluido el tema fundamental del desarrollo como proceso multidimensional.

Sin embargo, no es claro que el proceso político esté permitiendo abordar adecuadamente los desafíos que enfrenta el país para avanzar hacia una nueva fase de crecimiento alto acompañada de una profundización de su desarrollo (diversificación productiva, creación de nuevas ventajas, aseguramiento de la sustentabilidad del crecimiento, fortalecimiento del capital humano y social, etc.).

\section{LA OPORTUNIDAD CREADA POR EL TERREMOTO DEL 27 DE FEBRERO}

Al momento en que se produce el terremoto del 27 de febrero de 2010, faltaban menos de dos semanas para el cambio de Gobierno (Central), la renovación del Congreso Nacional (parcial en el Senado y completa de la Cámara baja) y Chile parecía haber sorteado de manera relativamente exitosa las amenazas macroeconómicas y financieras de la crisis internacional que comenzó en 2008. Sin embargo durante la campańa electoral, también se había evidenciado la necesidad de acelerar el paso en ciertos frentes o introducir rectificaciones de uno u otro signo. 
No parecía tener sentido volver a los niveles de desregulación y vulnerabilidad económica de comienzos de los ańos 1930 o inicios de los años 1980. Una cosa distinta es que se discuta el perfeccionamiento de la regulación y la eliminación de aquellas disposiciones o trámites que son innecesarios o que generan costos que exceden los beneficios que se pretende lograr. Tampoco parecía razonable perder las oportunidades de coordinación económica que son complementarias y consistentes con el funcionamiento de los mercados, por miedo a que se confunda con la "planificación centralizada". Es posible hacer prospectiva económica en serio, pensar el futuro con todos los actores económicos, públicos y privados, y establecer mecanismos que permitan sintonizar los esfuerzos que cada uno hace en su propio ámbito de desarrollo.

Todo ello parece haberse vuelto imprescindible luego de una catástrofe como la que ha generado el Terremoto, pero no surge como expectativa natural cuando se mira la forma en que se han abordado estos temas en la nueva administración, con poca convocatoria a los actores económicos, sociales y políticos distintos de aquellos más directamente vinculados al nuevo Gobierno. Esta es la queja de muchos municipios, de muchos actores regionales y de una variedad de actores económicos, sociales y políticos.

En Chile existe un compromiso abrumadoramente mayoritario y, por ello, difícilmente revocable, con los rasgos fundamentales de una estrategia de desarrollo que se fundamenta en el rol central de la iniciativa privada, los mercados libres y competitivos, las regulaciones efectivas, tecnificadas y transparentes, la importancia crucial de la orientación exportadora y la apertura externa en todos los campos. Pero también existe un grado de acuerdo abrumadoramente mayoritario sobre la necesidad de avanzar hacia una sociedad en la que todos sus miembros disfruten de la libertad económica y del bienestar que son consubstanciales a una convivencia civilizada. Por ello es fundamental poner fin a discusiones estériles sobre Mercado y Estado, instrumentos que es necesario utilizar de manera complementaria, en los ámbitos y con las precauciones que corresponde adoptar.

Hay una oportunidad, no sólo para reconstruir bien sino para aprovechar la coyuntura y abordar problemas pendientes en materia de desarrollo. Chile aprovechó oportunidades como esta en el pasado. No sería la primera vez. 


\section{REFERENCIAS BIBLIOGRAFÍAS}

Albala-Bertrand, J. M. (1993). "Natural disaster situations and growth: A macroeconomic model for sudden disaster impacts". World Development, Vol. 21, Nº, pp. 1417-1434.

Alesch, D. J. \& Petak, Wi. J. (1986).”The Politics and Economics of Earthquake Hazard Mitigation. Unreinforced Masonry Buildings in Southern California”, Monograph No43, Program on Environment and Behavior, University of Colorado.

Anbarci, N.; Escaleras, M. \& Register, C.A (2005). "Earthquake fatalities: the interaction of nature and political economy”. Journal of Public Economics Vol. 89 N$^{\circ}$ 9/10, pp. 1907-1933.

Barros, A. (2010). "El comportamiento de la infraestructura tecnológica y de comunicaciones. Dossier Información y Catástrofes", Cuadernos de Información №26, pp. 123-137.

BID (2007). Prevención y no solo respuesta a desastres, Documento complementario a la Política sobre gestión del riesgo de desastres. Washington: BID.

Díaz, J.; Lüders, R. \& Wagner, G. (2007). "Economía Chilena 1810-2000. Producto total y sectorial. Una nueva mirada”, Documento de Trabajo N³15, PUC: Instituto de Economía.

Escaleras, M.; Anbarci, N. \& Register, C.A. (2007). "Public sector corruption and major earthquakes: A potentially deadly interaction”. Public Choice N¹32, pp. 209-230.

Gallopín, G.C.; Gutman, P. \& Maletta, H. (1989). “Global Impoverishment, Sustainable Development and the Environment. A Conceptual Approach”. International Social Science Journal, Vol. 121, pp. 375-397.

Gallego, F. y Loayza, N. (2002). “The Golden Period for Growth in Chile: explanations and forecasts”. En Norman Loayza y Raimundo Soto (editores) Economic Growth: Sources, Trends and Cycles. Santiago: Serie de libros Banca Central, Análisis y Políticas Económicas.

Gallopín, G. C. (2003). "Una síntesis sistémica de las relaciones entre vulnerabilidad, amenaza, exposición e impacto, dirigida a la identificación de políticas". En CEPAL, Manual de Estimación de los Efectos Socioeconómico y Ambiental de los Desastres. Santiago: Comisión Económica para América Latina y el Caribe. http://www.eclac.cl/dmaah/mdn/cd/manual/esp/metodologia.pdf

Greenwald, B. C. \& Stiglitz, J. E. (1986). "Externalities in Economies with Imperfect Information and Incomplete Markets". Quarterly Journal of Economics N ${ }^{\circ}$ 90, pp. 229-264.

Kahn, M. E. (2003). The Death Toll from Natural Disasters: The Role of Income, Geography, and Institutions. Tufts University and Stanford University.

Kahn, M. E. (2005). "The death toll from natural disasters: the role of income, geography, and institutions". Review of Economics and Statistics Vol., 87, N², pp. 271-284. 
Keefer, P.; Neumayer, E. \& Plümper, T. (2010). "Earthquake Propensity and the Politics of Mortality Prevention”. Policy Research Working Paper 5182. The World Bank Development Research Group Macroeconomics and Growth Team \& Global Facility for Disaster Reduction and Recovery Department.

Kenny, Ch. (2009). "Why Do People Die in Earthquakes? The Costs, Benefits and Institutions of Disaster Risk Reduction in Developing Countries". Policy Research Working Paper 4823. The World Bank Sustainable Development Network Finance Economics \& Urban Department.

Kong, Laura S.L. (2010). “Tsunamis, una visión integral: ciencia, economía y educación”, Seminario de la American Academy of Science and Technology y el Campus Santiago de la Universidad de Talca, 29 de marzo de 2010.

Landerretche, O.A.; Lanzarotti, M. \& Ominami, C. (2004). "El Desarrollo Económico de Chile en la Encrucijada: o Cómo las Viejas Controversias Impiden Abordar los Nuevos Problemas". Revista Foro $\mathrm{N}^{\circ} 34$, pp. 9-20.

Loayza, N.; Olaberria, E.; Rigolini, J. \& Christiansen, L. (2009). "Natural Disasters and Growth Going beyond the Averages". Policy Research Working Paper 4980. The World Bank East Asia and Pacific Social Protection Unit \& Development Research Group.

Maloney, Wi. F. (2002). "Missed Opportunities: Innovation and Resource-Based Growth in Latin America”. Economia, Vol. 3, №1, pp. 111-151.

Ministerio de Hacienda de Chile (2010). Acta de resultados del Comité Consultivo del PIB tendencial. Santiago: Gobierno de Chile.

Nell, M. \& Richter, A. (2004). "Catastrophic Events as Threats to Society: Private and Public Risk Management Strategies”. Working Papers on risk and Insurance $\mathrm{N}^{\circ} 12$, Hamburg University.

Pinto, A. (1959). Chile, Un Caso de Desarrollo Frustrado. Santiago: Universitaria.

Stiglitz, J. E, (2009). "The Current Economic Crisis and Lessons for Economic Theory". Eastern Economic Journal, $\mathrm{N}^{\circ} 35$, pp. 281-296.

Wakker, P. P. (2008). Uncertainty. The New Palgrave Dictionary of Economics, Second Edition, Edited by Steven N. Durlauf and Lawrence E. Blume.

Yamamura, E. (2009). "Effects of interactions among social capital, income, and learning from experiences of natural disasters: A case study from Japan”. MPRA Paper No. 16223, posted 14. http://mpra.ub.uni-muenchen.de/16223/2/MPRA_paper_16223.pdf 